\title{
Conforto térmico e desempenho de bezerras Girolando alojadas em abrigos individuais com diferentes coberturas
}

\author{
Janice M. C. Barnabé ${ }^{1}$, Héliton Pandorfi², Gledson L. P. de Almeida², Cristiane Guiselini² \& Arthur L. Jacob ${ }^{3}$ \\ ${ }^{1}$ Programa de Pós-Grauação em Engenharia Agricola/ Universidade Federal Rural de Pernambuco. Recife, PE. E-mail: janice_coelho@yahoo.com.br \\ ${ }^{2}$ Departamento de Engenharia Agrícola/Universidade Federal Rural de Pernambuco. Recife, PE. E-mail: pandorfi@dtr.ufrpe.br (Autor correspondente); \\ gledson@dtr.ufrpe.br; cguiseli@hotmail.com \\ ${ }^{3}$ Engenharia Agrícola Ambiental/ Universidade Federal Rural de Pernambuco. Recife, PE. E-mail: arthurljacob@gmail.com
}

\section{Palavras-chave: ambiência animal bovinos de leite ganho de peso materiais de cobertura}

\begin{abstract}
R E S U M O
Esta pesquisa foi desenvolvida com o objetivo de caracterizar os efeitos do ambiente térmico nos índices de conforto, respostas fisiológicas e no desempenho de bezerras Girolando, alojadas em abrigos individuais cobertos com diferentes materiais. $\mathrm{O}$ experimento foi realizado entre janeiro e março de 2012, com duração de 56 dias, conduzido com 24 bezerras de composição genética 7/8 Holandês-Gir aos 15 dias de vida e com peso médio de 40,6 kg. Os tratamentos consistiram em três tipos de cobertura, palha de palmeira, telha de polímero reciclado e telha de fibrocimento, com 8 repetições. O delineamento experimental adotado foi o inteiramente casualizado com comparação entre médias pelo teste de Tukey $(\mathrm{p}<0,05)$. A entalpia e a carga térmica radiante diferiram estatisticamente em todos os tratamentos sendo os menores valores apresentados pelos abrigos com cobertura de telha reciclada, 59,3 $\mathrm{kJ} \mathrm{kg}^{-1}$ de ar seco e 444,8 W m${ }^{-2}$, respectivamente. Não houve diferença significativa em nenhuma das variáveis fisiológicas estudadas, porém a frequência respiratória esteve elevada em todos os tratamentos sendo mais acentuada nos animais sob cobertura de fibrocimento $\left(57,2 \mathrm{mov} \mathrm{min}^{-1}\right)$ indicando mais suscetibilidade ao estresse térmico.
\end{abstract}

\section{Key words:}

animal ambience

dairy cattle

weight gain

roofing materials

\section{Thermal comfort and performance Holstein/Gir calves housed in individual shelters with different covers}

\begin{abstract}
A B S T R A C T
This research was developed with the aim to characterize the effects of thermal ambience on physiological responses, comfort indexes and Holstein/Gir calves performance, housed in individual hutches covered with different materials. The experimental period was from January to March of 2012, lasting for 56 days, conducted by 24 crossbred calves with genetic of $7 / 8$ Holstein-Gir. The treatments consisted of three types of covering materials, palm straw, recycled polymer tile and fiber cement tile. A completely randomized design was adopted and in order to compare means the Tukey test $(\mathrm{p}<0.05)$ was used. The enthalpy and the radiant thermal load differed statistically in all treatments, being the lowest values presented by the recycled- tile-covered housings, $59.3 \mathrm{~kJ} \mathrm{~kg}^{-1}$ dry air and $444.8 \mathrm{~W} \mathrm{~m}^{-2}$, respectively. As for the physiological variables, there was no significant difference in any of the studied variables, however, the respiratory frequency in all treatments was high, and more emphasized on animals under fiber cement covered hutches $\left(57.2 \mathrm{mov} \mathrm{min}^{-1}\right)$ indicating that the animals were under heat stress.
\end{abstract}




\section{INTRODUÇÃo}

$\mathrm{O}$ ambiente térmico exerce forte influência sobre o desempenho animal uma vez que afeta os mecanismos de transferência de calor e, consequentemente, a regulação do balanço térmico entre o animal e o ambiente (Perissinoto \& Moura, 2007). Souza et al. (2010) afirmaram que, mesmo os animais com boa capacidade morfofisiológica para dissipar calor, necessitam de sombra natural ou artificial para se proteger da radiação solar direta, principalmente em regiões tropicais tendo em vista que a magnitude do estresse térmico é causada pelo efeito combinado da temperatura do ar, umidade relativa do ar, radiação solar e velocidade do vento (Dikmen \& Hansen, 2009).

Quando os mecanismos de termólise dos animais homeotérmicos não são suficientes para a manutenção da temperatura corporal, o calor metabólico, somado ao calor recebido do ambiente, torna-se maior que o calor dissipado promovendo o desequilíbrio térmico no animal. Desta forma, o organismo reage aumentando a sudorese e a frequência respiratória a fim de eliminar o excesso de calor (Morais et al., 2008).

O ganho de peso pode ser afetado pelas condições climáticas adversas ocasionando perdas na produção e na produtividade individual (Furtado et al., 2012). Assim, o entendimento das variações diárias e sazonais das respostas fisiológicas permite a adoção de ajustes que promovam maior conforto aos animais (Nóbrega et al., 2011).

Neste contexto, a pesquisa foi conduzida com o objetivo de caracterizar os efeitos do ambiente térmico nos índices de conforto, respostas fisiológicas e no desempenho de bezerras Girolando, alojadas em abrigos individuais cobertos com diferentes materiais.

\section{Material e Métodos}

A pesquisa foi realizada em uma fazenda de produção de leite localizada no município de Pesqueira, Mesorregião Agreste e Microrregião do Vale do Ipojuca, estado de Pernambuco ( $8^{\circ} 17^{\prime} 10^{\prime \prime} \mathrm{S} ; 36^{\circ} 53^{\prime} 3^{\prime \prime}$ W W e altitude de $800 \mathrm{~m}$ ). O clima do local é classificado, segundo Köeppen, como BShw' semiárido quente, caatinga hiperxerófila, com temperatura média anual de $27^{\circ} \mathrm{C}, 73 \%$ de umidade relativa do ar e $2,5 \mathrm{~m}$ $\mathrm{s}^{-1}$ de velocidade do vento (Silva et al., 2010).

O experimento foi conduzido durante o verão, entre janeiro a março de 2012, com duração de 56 dias. Foram utilizadas 24 bezerras Girolando em aleitamento, aos 15 dias de vida, com peso médio de 40,6 kg e composição genética 7/8 Holandês-Gir. As bezerras foram apartadas da mãe $24 \mathrm{~h}$ após o nascimento e alojadas em abrigos individuais. Até o terceiro dia de vida receberam colostro ( $\left.4 \mathrm{~L}_{\text {animal }}{ }^{-1} \mathrm{~d}^{-1}\right)$ fornecido duas vezes ao dia (manhã e tarde). A partir do quarto dia de vida as bezerras receberam leite integral ( $4 \mathrm{~L}$ animal $\left.^{-1} \mathrm{~d}^{-1}\right)$, também fracionados em duas refeições diárias (manhã e tarde). O desmame ocorreu aos 71 dias de idade, conforme manejo adotado pela unidade de produção.

A partir da primeira semana de vida foi oferecido, à vontade e em baldes individuais, o concentrado, contendo $18 \%$ de proteína bruta. Os animais tinham livre acesso à água.
Os tratamentos consistiram em três tipos de cobertura para os abrigos individuais: palha de palmeira Syagrus olearacea (Figura 1B), telha reciclada de $4 \mathrm{~mm}$ de espessura composta por $75 \%$ de polietileno e $25 \%$ de alumínio (Figura 1C) e telha de fibrocimento de $4 \mathrm{~mm}$ de espessura (Figura 1D). Os abrigos individuais possuíam dimensões de $1,8 \mathrm{~m}$ de comprimento, 1,5 m de largura e altura média de $1,45 \mathrm{~m}$, sem fechamento lateral, em uma água e cama de areia.
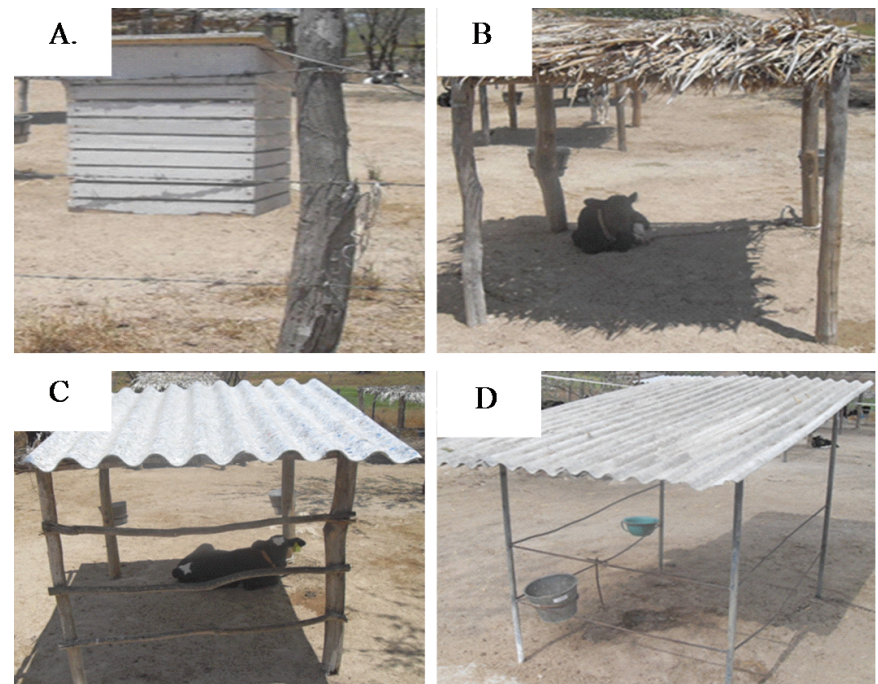

Figura 1. Abrigo meteorológico (A), bezerreiro coberto com palha, (B), telha reciclada (C) e telha de fibrocimento (D)

As variáveis meteorológicas foram medidas no interior de cada um dos abrigos individuais e no abrigo meteorológico (Figura 1A), por meio de gravador de dados modelo HOBO U12-12, o que permitiu o registro automático da temperatura de bulbo seco Tbs $\left({ }^{\circ} \mathrm{C}\right)$, da umidade relativa do ar UR (\%) e da temperatura de globo negro $\operatorname{Tg}\left({ }^{\circ} \mathrm{C}\right)$, no decorrer de todo o período experimental. Os sensores dos abrigos dos animais foram posicionados no centro geométrico da instalação a $1,4 \mathrm{~m}$ do piso a fim de evitar o contato dos animais com os mesmos. O abrigo meteorológico foi instalado a $1,50 \mathrm{~m}$ de altura do solo. Para a determinação da velocidade do vento $\mathrm{Vv}\left(\mathrm{m} \mathrm{s}^{-1}\right)$ utilizou-se um termoanemômetro digital, modelo TAFR-180, escala de 0,1 a $20,0 \mathrm{~m} \mathrm{~s}^{-1}$ e resolução de $0,1 \mathrm{~m} \mathrm{~s}^{-1}$. Todos os registros foram realizados a cada hora, durante todo o período de avaliação.

Para a análise térmica das instalações foram determinados a carga térmica radiante CTR ( $\mathrm{W} \mathrm{m}^{-2}$ ), proposta por Esmay (1982), o índice de temperatura e umidade ITU, proposto por Thom (1959), o índice de temperatura de globo e umidade ITGU, proposto por Buffington et al. (1981) e a entalpia específica $\mathrm{h}\left(\mathrm{kJ} \mathrm{kg}^{-1}\right.$ de ar seco) sugerida por Rodrigues et al. (2010a) por meio das equações 1, 2, 3, 4 e 5:

$$
\begin{gathered}
\mathrm{CTR}=\sigma(\mathrm{TRM})^{4} \\
\operatorname{TRM}=100\left\{\left[2,51\left(\mathrm{~V}_{\mathrm{v}}\right)^{0,5}\left(\mathrm{~T}_{\mathrm{gn}}-\mathrm{T}_{\mathrm{bs}}\right)+\left(\frac{\mathrm{T}_{\mathrm{gn}}}{100}\right)^{4}\right]^{0,25}\right\}
\end{gathered}
$$

em que:

TRM - temperatura média radiante, $\mathrm{K}$ 
Vv - velocidade do vento, $\mathrm{m} \mathrm{s}^{-1}$

Tgn - temperatura de globo negro, $\mathrm{K}$

Tbs - temperatura de bulbo seco, $\mathrm{K}$;

$\sigma=5,67 * 10^{-8} \mathrm{~K}^{-4} \mathrm{~W} \mathrm{~m} \mathrm{~m}^{-2}$ (Constante de StefanBoltzmann)

$$
\mathrm{ITU}=\mathrm{T}_{\mathrm{bs}}+0,36 \cdot \mathrm{T}_{\mathrm{po}}+41,5
$$

em que:

Tbs - temperatura de bulbo seco, ${ }^{\circ} \mathrm{C}$

Tpo - temperatura de ponto de orvalho, ${ }^{\circ} \mathrm{C}$

$$
\mathrm{ITGU}=\mathrm{Tgn}+0,36 \mathrm{Tpo}+330,08
$$

em que:

Tgn - temperatura de globo negro, $\mathrm{K}$

Tpo - temperatura de ponto de orvalho, $\mathrm{K}$

$$
\left.\mathrm{h}=1,006 \cdot \mathrm{T}_{\mathrm{bs}}+\frac{\mathrm{UR}}{\mathrm{P}_{\mathrm{atm}}} \cdot 10^{\left(\frac{7,5 \cdot \mathrm{T}_{\mathrm{bs}}}{237,3}+\mathrm{T}_{\mathrm{bs}}\right.}\right) \cdot\left(71,28+0,052 \cdot \mathrm{T}_{\mathrm{bs}}\right)
$$

em que:

Tbs - temperatura de bulbo seco, ${ }^{\circ} \mathrm{C}$

UR - umidade relativa, \%

Patm - pressão atmosférica do local, $\mathrm{mmHg}$

Para avaliação dos parâmetros fisiológicos foram registrados, em todos os animais de todos os tratamentos: temperatura retal TR $\left({ }^{\circ} \mathrm{C}\right.$ ), frequência respiratória FR (mov $\left.\mathrm{min}^{-1}\right)$ e temperatura de pelame $\mathrm{TP}\left({ }^{\circ} \mathrm{C}\right)$, realizados nos horários das $5,8,11,14$ e $17 \mathrm{~h}$, duas vezes por semana, durante todo o experimento.

A verificação da FR deu-se a partir da contagem do número de movimentos da região do flanco realizados pelo animal, no intervalo de 1 min segundo metodologia adotada por MacLean (2012). Após o registro da FR foram tomadas as medidas da TR, com auxílio de termômetro digital de uso veterinário com escala entre 20 e $50{ }^{\circ} \mathrm{C}$ e precisão de $\pm 0,1^{\circ} \mathrm{C}$.

Para determinação da TP foram utilizadas imagens térmicas obtidas por meio de uma câmera termográfica, modelo FLIR i60. As imagens foram registradas do lado esquerdo dos animais e se adotou uma distância padrão de 2,5 m entre a câmera e o animal.

As análises das imagens termográficas foram realizadas pelo programa computacional FLIR QuickReport, em que se ajustou o valor da emissividade para 0,95 conforme utilizado por Moura et al. (2011) em experimento com equinos. As variáveis termo-higrométricas, temperatura e umidade relativa do ar, foram obtidas no instante do registro da imagem; assim, delimitou-se uma área que compreendeu a região do tórax e abdômen dos animais obtendo-se uma superfície representativa do corpo do indivíduo (Figura 2).

O desempenho dos animais foi avaliado por meio do ganho de peso GP $(\mathrm{kg})$, em que os indivíduos foram pesados no primeiro e no último dia do período experimental, por meio de balança eletrônica, modelo ID-M 300/4, acoplada a uma estrutura de contenção.

Cada tratamento contou com oito repetições utilizando-se delineamento inteiramente casualizado. A análise estatística das

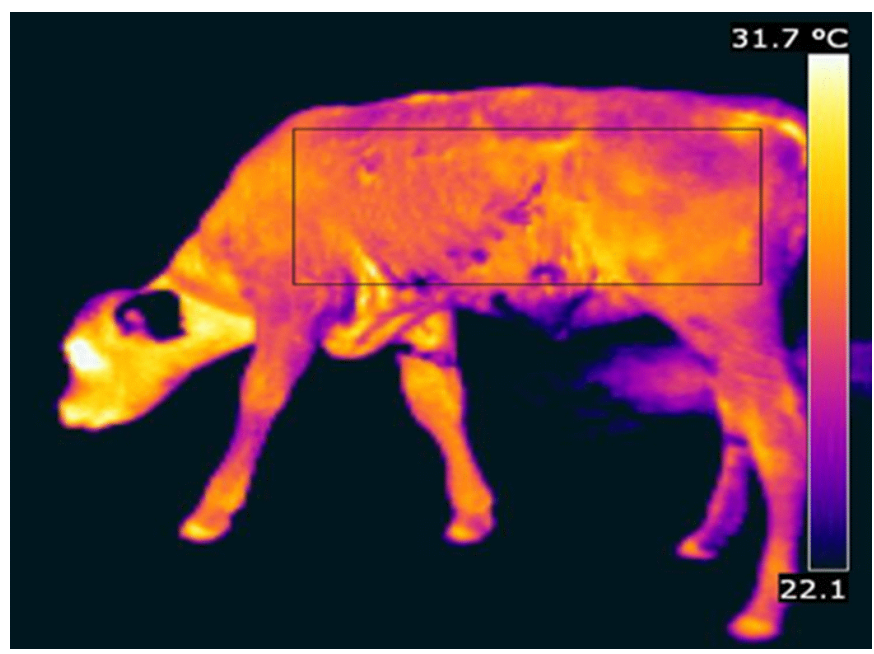

Figura 2. Imagem térmica da superfície do animal obtida a partir do registro da câmera termográfica, para estimativa da temperatura de pelame $\left({ }^{\circ} \mathrm{C}\right)$

variáveis fisiológicas, dos índices de conforto e do desempenho foi realizada por meio do programa computacional Statistcal Analysis System (SAS, 2007) e as médias comparadas pelo teste de Tukey $(\mathrm{p}<0,05)$. Para avaliação das variáveis ambientais foram realizadas análises de regressão.

\section{Resultados e Discussão}

Observa-se, na Figura 3A, variação similar da temperatura média do ar no interior dos abrigos e no abrigo meteorológico em que o abrigo coberto com telhas de fibrocimento apresentou temperaturas mais elevadas entre os horários das 8 às $14 \mathrm{~h}$.

A Figura 3B demonstra a relação funcional entre a temperatura do abrigo meteorológico e no interior do abrigo coberto com telhas de fibrocimento, que apresentou coeficiente de determinação da ordem de 0,9395, o que denota boa associação linear entre os valores; verificou-se, portanto, que a temperatura interna do abrigo foi $0,68 \%$ maior do que no abrigo meteorológico.

A relação entre a temperatura do abrigo com cobertura de palha e o abrigo meteorológico aponta variação de $0,58 \%$ superior àquela que se verifica no abrigo meteorológico (Figura 3C). Os abrigos com cobertura de telhas recicladas apresentaram temperatura interior menor $(0,79 \%)$ que a do abrigo meteorológico (Figura 3D).

A Figura 4A representa a variação da umidade relativa do ar no interior dos abrigos e no abrigo meteorológico. Verificase que todos os abrigos apresentaram valores bem próximos com destaque para as pequenas diferenças dos abrigos com telhas de fibrocimento e telhas recicladas que reduziram sua umidade relativa mais rapidamente.

A Figura 4B apresenta a relação entre a umidade relativa do ar no abrigo meteorológico e no interior do abrigo coberto com telhas de fibrocimento; nota-se que a umidade relativa do ar no interior do abrigo foi $0,47 \%$ maior do que no abrigo meteorológico.

Para o abrigo com cobertura de palha a umidade relativa do ar no interior do abrigo foi $1,05 \%$ maior que no abrigo meteorológico (Figura 4C); já nos abrigos cobertos por telhas 

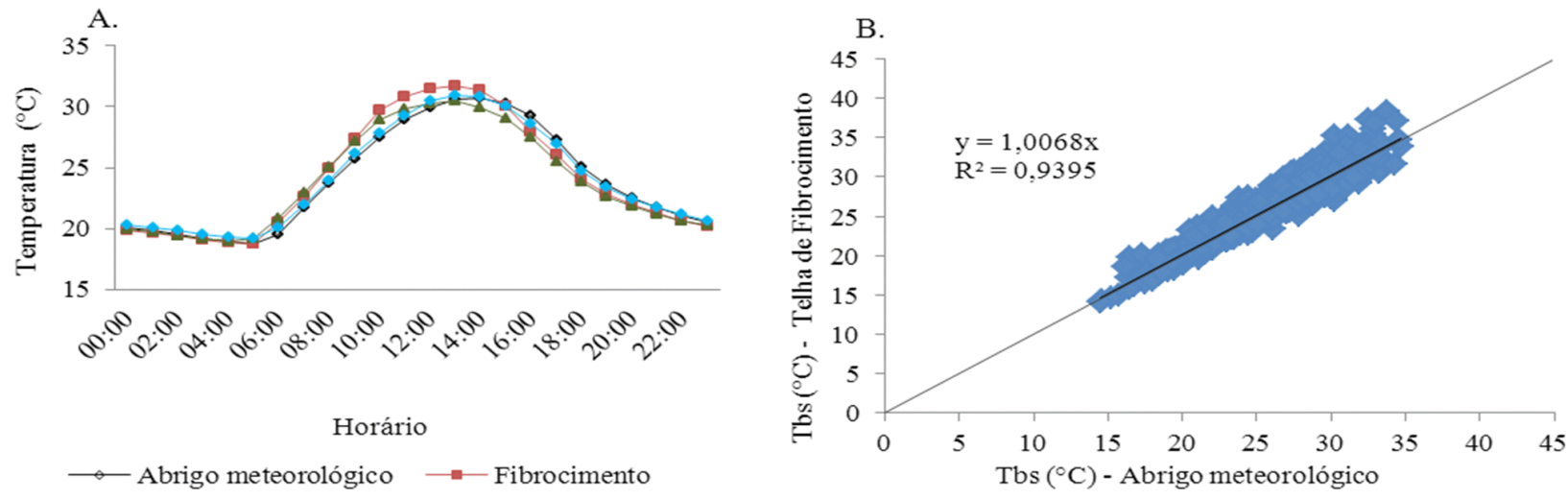

$\begin{array}{ll}\multimap \text { Abrigo meteorológico } & - \text { Fibrocimento } \\ \multimap \text { Reciclada } & \longrightarrow \text { Palha }\end{array}$
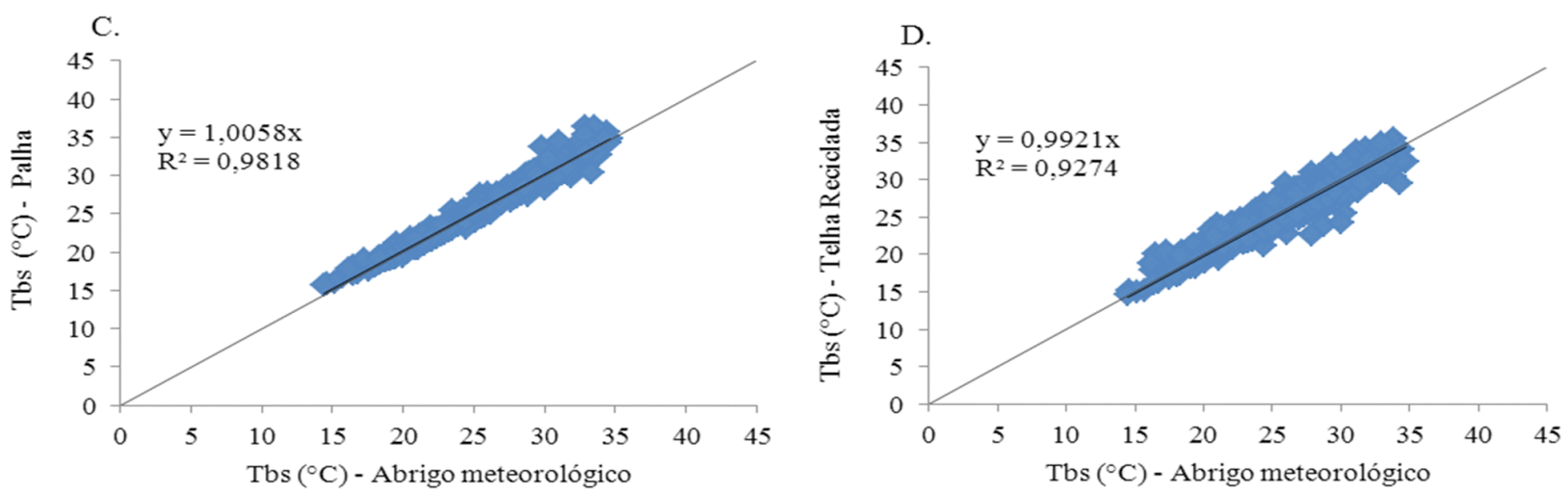

Figura 3. Variação média do abrigo meteorológico e relação funcional entre a temperatura do abrigo meteorológico e a temperatura no interior dos abrigos cobertos com: (B) telha de fibrocimento, $(C)$ palha e $(D)$ telha reciclada $(F<0,01)$
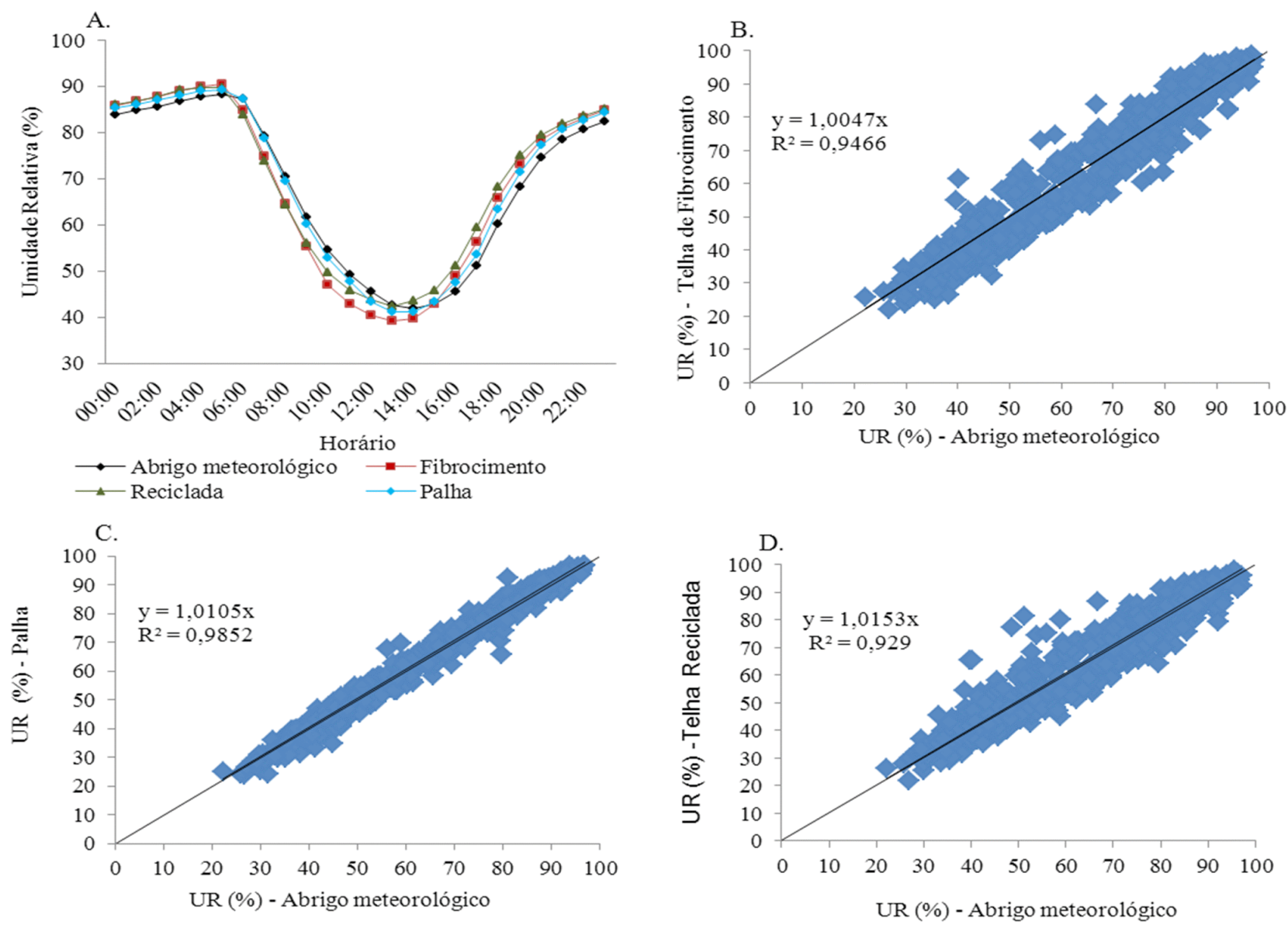

Figura 4. Variação média da umidade relativa (A) no interior dos abrigos nos diferentes tratamentos e no abrigo meteorológico e relação entre a umidade relativa (UR) do ambiente externo no interior dos abrigos com cobertos com: (B) telha de fibrocimento, $(C)$ palha e $(D)$ telha reciclada $(F<0,01)$ 
recicladas apresentaram umidade relativa do ar $1,53 \%$ maior que o abrigo meteorológico (Figura 4D).

Baêta \& Souza (2010) estabelecem os limites de temperatura do ar para bezerros de 10,18 a 21 e $26^{\circ} \mathrm{C}$, para temperatura crítica inferior TCI $\left({ }^{\circ} \mathrm{C}\right)$, zona de conforto térmico ZCT $\left({ }^{\circ} \mathrm{C}\right)$ e temperatura crítica superior TCS $\left({ }^{\circ} \mathrm{C}\right)$, respectivamente; desta forma, para evidenciar o efeito do acondicionamento térmico dos animais considerou-se o dia de maior e menor entalpia, sendo a entalpia crítica superior para bezerros de $66 \mathrm{~kJ} \mathrm{~kg}^{-1}$ de ar seco (Kawabata et al., 2005).

Observa-se, na Figura 5A, a variação diária da temperatura de bulbo seco $\left({ }^{\circ} \mathrm{C}\right)$ para o dia de maior entalpia (20 de fevereiro de 2012), que apresentou média de 62,75 $\mathrm{kJ} \mathrm{kg}^{-1}$ ar seco, indicando que, em seus respectivos abrigos, todos os animais abrigos estiveram dentro da ZCT das 21 às $6 \mathrm{~h}$ e fora deste intervalo se utilizaram de seus mecanismos termorregulatórios a fim de manter a homeotermia. Este valor foi inferior ao encontrado por Conceição et al. (2008) que verificaram valor médio da ordem de 70,23 $\mathrm{kJ} \mathrm{kg}^{-1}$ ar seco, em estudo utilizando cobertura de telhas convencionais e composta à base de fibra vegetal. A partir das $8 \mathrm{~h}$ a temperatura no interior dos abrigos ultrapassou até 6,9 e $5,3{ }^{\circ} \mathrm{C}$ a temperatura TCS, para coberturas de telhas de fibrocimento e recicladas, respectivamente.

Os animais alojados em abrigos com cobertura de telhas de fibrocimento se mantiveram com temperatura acima da TCS até as $14 \mathrm{~h}$. Os abrigos com cobertura de telhas recicladas reduziram a temperatura do ar mais rapidamente que os demais. Neste dia de maior entalpia a temperatura esteve acima da ZCT para bezerras, por um período de aproximadamente $16 \mathrm{~h}$ diárias (6 às $21 \mathrm{~h}$ ), sendo que esses animais permaneceram durante $6 \mathrm{~h}$ ( 9 às $13 \mathrm{~h}$ ) deste dia, sujeitos ao desconforto térmico, ou seja, em temperatura acima da TCS (Figura 5A).

Nos abrigos cobertos com palha constatou-se pequeno retardo para ultrapassar a TCS quando comparado com as
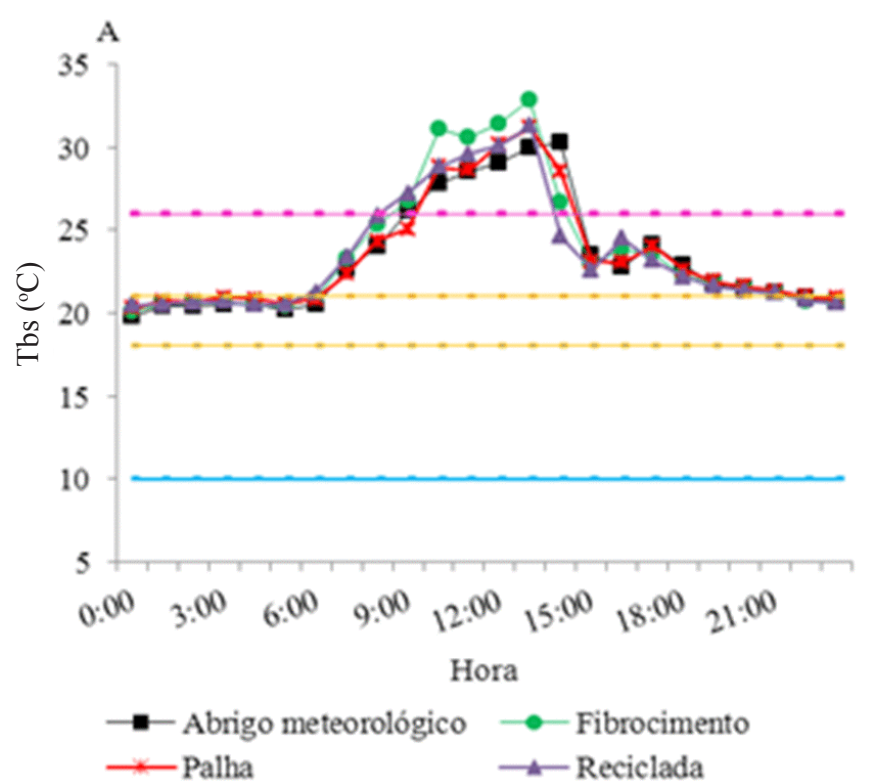

Figura 5. Variação média diária da temperatura de bulbo seco (Tbs, $\left.{ }^{\circ} \mathrm{C}\right)$ no interior dos abrigos nos diferentes tratamentos e no abrigo meteorológico nos dias de maior (A) e menor entalpia (B), temperatura crítica inferior (TCI), zona de conforto térmico (ZCT) e temperatura crítica superior (TCS) para bezerros outras coberturas (Figura 5A). Isso ocorreu por se tratar de um material orgânico e fibroso, que absorve a água provinda da condensação na superfície da cobertura no período noturno. Desta forma, parte da energia gasta para aquecer o material foi utilizada para evaporar a água absorvida pela cobertura de palha, portanto, só após ter ocorrido este processo o material voltou a se aquecer retardando a transferência de calor para o interior da instalação condição em que o valor máximo alcançado acima da TCS foi de $5,2{ }^{\circ} \mathrm{C}$.

Para o dia de menor entalpia (16 de janeiro de 2012), verificou-se valor médio da ordem de $52,84 \mathrm{~kJ} \mathrm{~kg}^{-1}$ ar seco. A temperatura do ar apresentou, em todos os abrigos, comportamento térmico bem semelhante. Os animais estiveram dentro da ZCT entre 0 e $6 \mathrm{~h}$ e das 20 às $23 \mathrm{~h}$. A TCS foi ultrapassada entre as 9 e $17 \mathrm{~h}$, período em que as bezerras sofreram estresse por calor (Figura $5 \mathrm{~B}$ ).

Nota-se que tanto nos dias de maior entalpia (Figura 5A) como de menor entalpia (Figura 5B) os animais estiveram em condições de desconforto térmico na maior parte do dia. Segundo Rodrigues et al. (2010b) a intensidade e o tempo de permanência a que os bovinos leiteiros estão submetidos ao estresse térmico, conduzem as consequências de tal estresse por um período superior àquele em que foram expostos.

O ITU apontou efeito significativo $(\mathrm{p}<0,05)$ para o abrigo com cobertura de palha em comparação aos demais tipos de cobertura e para o abrigo meteorológico, com valor da ordem de 76,3 ultrapassando o limite máximo de conforto térmico suportado pelo animal, sem haver necessidade de dispêndio de energia para o sistema de termorregulação, que é de 72 (Hanh, 1982).

Os valores de ITU para as coberturas de fibrocimento e reciclada foram de 72,0 e 71,6, respectivamente (Tabela 1). Estão próximos aos observados por Conceição et al. (2008), que verificaram valores de ITU de 72,18 para telha composta, 72,29 para telha cerâmica e 71,97 para telha de fibrocimento.

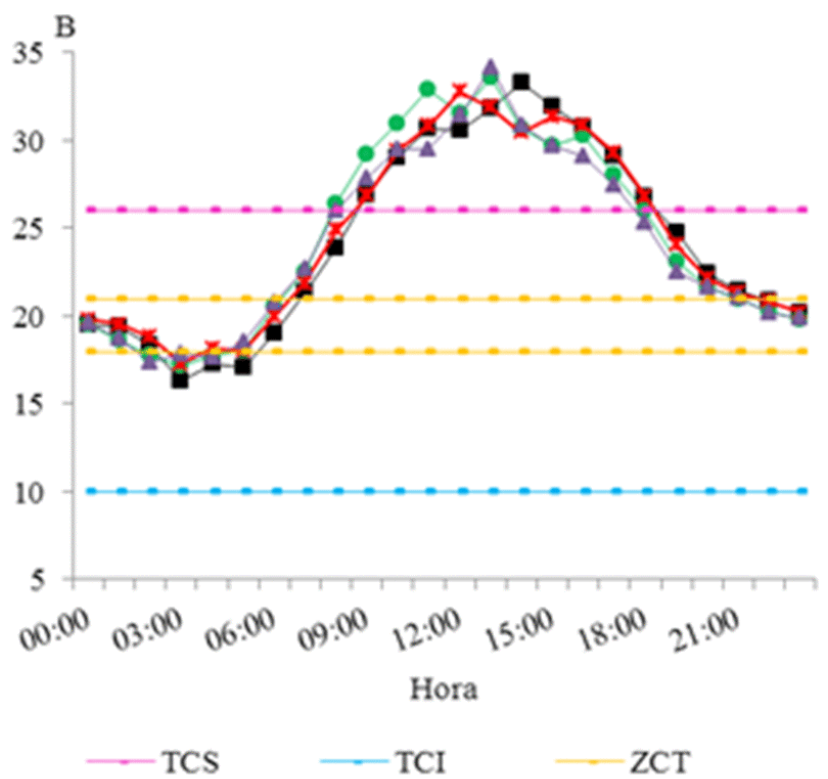


Tabela 1. Valores médios, desvio padrão e coeficiente de variação dos índices de conforto térmico obtidos no interior dos abrigos e no abrigo meteorológico

\begin{tabular}{lccccc}
\hline \multirow{2}{*}{ Variáveis } & \multicolumn{2}{c}{ Tratamentos } & PV \\
\cline { 2 - 5 } ITU & Abrigo meteorológico & Fibrocimento & Palha & Reciclada & (\%) \\
ITGU & $71,8 \mathrm{~b} \pm 0,13$ & $72,0 \mathrm{~b} \pm 0,22$ & $76,3 \mathrm{a} \pm 6,10$ & $71,6 \mathrm{~b} \pm 0,05$ & 4,84 \\
$\mathrm{~h}\left(\mathrm{~kJ} \mathrm{~kg}^{-1} \mathrm{ar} \mathrm{sec0)}\right.$ & $73,9 \mathrm{a} \pm 0,05$ & $72,9 \mathrm{a} \pm 0,26$ & $73,9 \mathrm{a} \pm 4,13$ & $71,9 \mathrm{a} \pm 0,03$ & 2,93 \\
CTR $\left(\mathrm{W} \mathrm{m}{ }^{-2}\right)$ & $58,9 \mathrm{~d} \pm 0,04$ & $59,6 \mathrm{~b} \pm 0,05$ & $59,7 \mathrm{a} \pm 0,05$ & $59,3 \mathrm{c} \pm 0,04$ & 0,49 \\
\hline
\end{tabular}

Médias seguidas das mesmas letras nas mesmas linhas não diferem entre si a nível de 0,05 de probabilidade, pelo teste de Tukey. ITU - Índice de temperatura e umidade; ITGU - Índice de temperatura de globo e umidade; $\mathrm{h}$ - Entalpia específica; CTR - Carga térmica de radiação

Não houve diferença estatística $(\mathrm{p}>0,05)$ entre os tipos de cobertura e o ambiente externo para o ITGU; no entanto e de acordo com Baêta \& Souza (2010) os valores verificados (Tabela 1) indicam condição de conforto (ITGU < 74) para os bovinos, proporcionado pelo efeito do sombreamento promovido pela cobertura. O valor do ITGU obtido no abrigo com cobertura de fibrocimento está dentro do intervalo encontrado por Sampaio et al. (2011) entre 64,2 e 73,5 e menores que os obtidos por Fonseca et al. (2011) que avaliaram coberturas de telha de zinco $(81,63)$; telha de cimento amianto $(77,41)$ e telha de cimento amianto pintada de branco na face superior $(76,84)$, em abrigos individuais para bezerros leiteiros.

Embora tenha apresentado valores bem próximos, a variável entalpia apresentou diferença significativa $(p<0,05)$ no interior do abrigo meteorológico e no interior dos abrigos dos animais, sendo eles: 58,9; 59,6; 59,7 e 59,3 $\mathrm{kJ} \mathrm{kg}^{-1}$ de ar seco para o abrigo meteorológico, abrigo com cobertura de fibrocimento, cobertura de palha e telha reciclada, respectivamente (Tabela 1). Tais valores foram inferiores aos encontrados por Fonseca et al. (2011) que verificaram valor mínimo de 63,94 e máximo de $70,38 \mathrm{~kJ} \mathrm{~kg}^{-1}$ ar seco, principalmente pela baixa umidade relativa do ar entre as 10 e $16 \mathrm{~h}$ (Figura $4 \mathrm{~A}$ ).

$\mathrm{Na}$ avaliação da CTR verificou-se que todos os tratamentos e o abrigo meteorológico apresentaram diferença significativa $(\mathrm{p}<0,05)$, em que o abrigo coberto com telha reciclada apresentou melhor desempenho e o de telha de fibrocimento o pior, sendo que todos foram melhores que o abrigo meteorologico (Tabela 1). Cardoso et al. (2011) mostraram que as coberturas utilizadas em instalações para produção animal, atenuam a CTR exterior sobre os animais nos períodos críticos do dia e citam, como exemplo, que próximo às $13 \mathrm{~h}$ a redução ou amortecimento da CTR pode chegar entre 37 a 40\%, dependendo do tipo de material utilizado.

Os resultados de CTR obtidos no presente estudo foram semelhantes aos encontrados por Sampaio et al. (2011), que verificaram valores entre 406,7 a 479,$2 ; 405,0$ a 480,52 e 406,2 a $518,3 \mathrm{~W} \mathrm{~m}^{-2}$, para telhas cerâmicas, fibrocimento e metálicas, respectivamente. Fiorelli et al. (2010) avaliaram, durante o inverno, protótipos de galpões com diferentes materiais de cobertura a diferentes alturas $(0,30$ e $0,70 \mathrm{~m})$ do solo e obtiveram média diária para CTR de $525,01 \mathrm{~W} \mathrm{~m}^{-2}$ para cobertura de telhas fibrocimento e de $527,01 \mathrm{~W} \mathrm{~m}^{-2}$ para cobertura de telhas recicladas.

Os parâmetros fisiológicos e o ganho de peso não apontaram efeito significativo $(p>0,05)$ entre os animais criados nos abrigos individuais com diferentes materiais de cobertura (Tabela 2).

Em todos os abrigos (Tabela 2) a frequência respiratória (FR) dos animais se encontra acima da faixa normal
Tabela 2 Valores médios, desvio padrão e coeficiente de variação das variáveis fisiológicas e ganho de peso dos animais

\begin{tabular}{lcccc}
\hline \multirow{2}{*}{ Variáveis } & \multicolumn{3}{c}{ Tratamentos } & CV \\
\cline { 2 - 4 } & Fibrocimento & Palha & Reciclada & $(\%)$ \\
FR $\left({\left.\text { mov } \text { min }^{-1}\right)}^{57,2} \mathrm{a} \pm 6,61\right.$ & $53,5 \mathrm{a} \pm 5,97$ & $54,4 \mathrm{a} \pm 2,48$ & 9,73 \\
TR $\left({ }^{\circ} \mathrm{C}\right)$ & $39,0 \mathrm{a} \pm 0,29$ & $38,8 \mathrm{a} \pm 0,18$ & $38,9 \mathrm{a} \pm 0,28$ & 0,64 \\
TP $\left({ }^{\circ} \mathrm{C}\right)$ & $32,1 \mathrm{a} \pm 1,33$ & $32,6 \mathrm{a} \pm 0,49$ & $32,0 \mathrm{a} \pm 0,94$ & 3,02 \\
GP $(\mathrm{Kg})$ & $34,7 \mathrm{a} \pm 1,33$ & $32,6 \mathrm{a} \pm 7,88$ & $39,0 \mathrm{a} \pm 14,58$ & 33,82 \\
\hline
\end{tabular}

Médias seguidas das mesmas letras nas mesmas linhas não diferem entre si a nível de 0,05 de probabilidade, pelo teste de Tukey. FR - Frequência respiratória; TR - Temperatura retal; TP - Temperatura de pelame; GP - Ganho de peso

recomendada por Reece (2006) que é de 21 a $25 \mathrm{mov} \mathrm{min}^{-1}$, para bovinos de até um ano de idade. Mac-Lean (2012) avaliou a FR de bezerros da raça Jersey, pura e mestiça, alojados em cabanas individuais, cobertas com telha de fibrocimento e verificou valores que variaram de 27 a $80 \mathrm{mov} \mathrm{min}^{-1}$, apresentando-se como indicador de estresse térmico.

O primeiro sinal visível de animais submetidos ao estresse térmico é o aumento da FR. Portanto, este é um sinal de que os animais intensificaram os processos latentes de perda de calor na tentativa de manter a temperatura corporal dentro da normalidade fisiológica (Cunha et al., 2007). Os valores da temperatura retal (TR) variaram de 38,8 a $39,0^{\circ} \mathrm{C}$ (Tabela 2); portanto, permaneceram dentro do intervalo considerado normal para rebanhos leiteiros que, de acordo com Robertshaw (2006) podem variar de 38,0 a $39,3{ }^{\circ} \mathrm{C}$. A manutenção da TR dentro da faixa de normalidade pode ser justificada pelo mecanismo fisiológico de aumento da FR. Em todos os tratamentos a média da TR foi superior à encontrada por Mac-Lean (2012) que foi de 38,1 para os animais alojados em cabanas cobertas com telhas de fibrocimento.

Em seu estudo, Furtado et al. (2012) encontraram, com tourinhos das raças Sindi e Guzerá mantidos em regime de semiconfinamento, no Agreste paraibano, valores médios diários de 38,89 e $38,88^{\circ} \mathrm{C}$ de TR; 23,57 e 23,65 mov min $^{-1}$ de FR e 30,74 e $30,58^{\circ} \mathrm{C}$ de temperatura de pelame (TP), para as respectivas raças; portanto, todos os valores obtidos, exceto a TR, para o abrigo com cobertura de palha, se mantiveram acima dos encontrados por esses pesquisadores.

A menor média da temperatura de pelame (TP) deu-se para os animais alojados sob cobertura de telha reciclada (Tabela 2) cuja ocorrência pode ser devida às propriedades físicas do material as quais ampliam a capacidade de refletir a radiação solar. Do mesmo modo, Fonseca et al. (2012) afirmam que os animais mantidos sob cobertura de zinco estiveram em estágio de baixo estresse e justificam que isto pode ter ocorrido devido à diferença no balanço de radiação de ondas longas em relação aos demais materiais e à alta capacidade que o zinco tem de reflexão e dissipação do calor. 
Oliveira et al. (2012) avaliaram o efeito das variáveis meteorológicas sobre as respostas horárias dos parâmetros fisiológicos de novilhos da raça Sindi, distribuídos em baias individuais cobertas com tela de polietileno preta, com capacidade de retenção de iluminação de $70 \%$, em Petrolina$\mathrm{PE}$, local de condições climáticas semelhantes às do presente estudo e obtiveram valores médios de 23,2 mov min $^{-1}$ para $\mathrm{FR}, 32,8^{\circ} \mathrm{C}$ para $\mathrm{TP}$ e $38,88^{\circ} \mathrm{C}$ para TR.

Não houve diferença significativa $(p>0,05)$ para a variável GP das bezerras (Tabela 2), o que reforça os resultados apontados por Fonseca et al. (2012) que não observaram comprometimento no desempenho dos animais em relação aos manejos de cobertura avaliados, cimento amianto pintada de branco, zinco galvanizado e cimento amianto.

\section{Conclusões}

1. A análise dos índices de conforto ressaltou que os abrigos cobertos com telhas recicladas apresentam melhor desempenho térmico.

2. Quanto às variáveis fisiológicas e independentemente dos tipos de cobertura utilizados nos abrigos individuais, a frequência respiratória esteve acima da faixa normal para bovinos naquela idade o que sinalizou que os mesmos estavam submetidos a estresse térmico, porém referido mecanismo foi capaz de manter a temperatura retal dentro dos limites normais.

3. As coberturas de telha reciclada, telha de fibrocimento e palha, utilizadas nos abrigos individuais não influenciaram no ganho de peso das bezerras em fase de aleitamento.

\section{Literatura Citada}

Baêta, F. C.; Souza, C. F. Ambiência em edificações rurais - Conforto animal. 2.ed. Viçosa: UFV, 2010. 246p.

Buffington, D. E.; Collasso-Arocho, A.; Canton, G. H.; Pit, D. Black globe-humidity index (BGHI) as comfort equation for dairy cows. Transactions of the ASAE, v.24, p.711-714. 1981. http://dx.doi. org/10.13031/2013.34325

Cardoso, A. S.; Baêta, F. C.; Tinôco, I. F. F.; Cardoso, V. A. S. Coberturas com materiais alternativos de instalações de produção animal com vistas ao conforto térmico. Engenharia na Agricultura, v.19, p.404421, 2011. http://dx.doi.org/10.13083/1414-3984.v19n05a02

Conceição, M. N.; Alves, S. P.; Telatin Júnior, A.; Silva, I. J. O.; Piedade, S. M. S.; Savastano Júnior, H.; Tonoli, G. Desempenho de telhas de escória de alto forno e fibras vegetais em protótipos de galpões. Revista Brasileira de Engenharia Agrícola e Ambiental, v.12, p.536539, 2008. http://dx.doi.org/10.1590/S1415-43662008000500015

Cunha, D. N. F. V.; Campos, O. F.; Pereira, J. C.; Pires, M. F. A.; Oliveira, R. F. M.; Martuscello, J. A. Desempenho, variáveis fisiológicas e comportamento de bezerros mantidos em diferentes instalações: Época seca. Revista Brasileira de Zootecnia, v.36, p.847-854, 2007. http://dx.doi.org/10.1590/S1516-35982007000400014

Dikmen, S.; Hansen, P. J. Is the temperature-humidity index the best indicator of heat stress in lactating dairy cows in a subtropical environment? Journal of Dairy Science, v.92, p.109-116, 2009. http://dx.doi.org/10.3168/jds.2008-1370

Esmay, M. L. Principles of animal environment. Westport: Avi, 1982. $325 \mathrm{p}$.
Fiorelli, J.; Fonseca, R.; Morceli, J. A.; Dias, A. A. Influência de diferentes materiais de cobertura no conforto térmico de instalações para frangos de corte no oeste paulista. Engenharia Agrícola, v.30, p.986-992, 2010. http://dx.doi.org/10.1590/S010069162010000500020

Fonseca, P. C. F.; Almeida, E. A.; Passini, R. Thermal comfort indexes in individual shelters for dairy calves with different types of roofs. Engenharia Agrícola, v.31, p.1044-1051, 2011. http://dx.doi. org/10.1590/S0100-69162011000600002

Fonseca, P. C. F.; Almeida, E. A.; Passini, R. Evaluation of covering materials in individual shelters and its effects on physiological responses and performance of dairy calves. Engenharia Agrícola, v.32, p.1041-1047, 2012. http://dx.doi.org/10.1590/S010069162012000600005

Furtado, D. A.; Peixoto, A. P.; Regis, J. E. F.; Nascimento, J. W. B. do; Araujo, T. G. P.; Lisboa, A. C. Termorregulação e desempenho de tourinhos Sindi e Guzerá, no Agreste paraibano. Revista Brasileira de Engenharia Agrícola e Ambiental, v.16, p.1022-1028, 2012. http://dx.doi.org/10.1590/S1415-43662012000900014

Hanh, G. L. Compensatory performance in livestock: Influence on environmental criteria. In: Livestock Environment, 2, 1982. Proceeding of the International Livestock Environment Symposium, 2, St. Joseph: ASAE, 1982. p.285-294.

Kawabata, C. Y.; Castro, R. C.; Savastano Júnior, H. Índices de conforto térmico e respostas fisiológicas de bezerros da raça holandesa em bezerreiros individuais com diferentes coberturas. Engenharia Agrícola, v.25, p.598-607, 2005. http://dx.doi.org/10.1590/S010069162005000300004

Mac-Lean, P. A. B. Programa de suplementação de luz e relações entre variáveis fisiológicas e termográficas de bezerros em aleitamento em clima quente. Pirassununga: USP, 2012. 103p. Tese Doutorado

Morais, D. A. E. F.; Maia A. A. C; Silva R. G.; Vasconcelos A. M.; Lima P. O.; Guilhermino, M. M. Variação anual de hormônios tireoideanos e características termorreguladoras de vacas leiteiras em ambiente quente. Revista Brasileira de Zootecnia, v.37, p.538545, 2008. http://dx.doi.org/10.1590/S1516-35982008000300020

Moura, D. J.; Maia, A. P. A.; Vercellino, R. A.; Medeiros, B. B. L.; Sarubbi, J.; Griska, P. R. Uso da termografia infravermelha na análise da termorregulação de cavalo em treinamento. Engenharia Agrícola, v.31, p.23-32, 2011. http://dx.doi.org/10.1590/S010069162011000100003

Nóbrega, G. H.; Silva, E. M. N.; Souza, B. B.; Mangueira, J. M. A produção animal sob a influência do ambiente nas condições do semiárido nordestino. Revista Verde de Agroecologia e Desenvolvimento Sustentável, v.6, p.67-73, 2011.

Oliveira, P. T. L.; Turco, S. H. N.; Araújo, G. G. L.; Voltolini, T. V.; Menezes, D. R.; Silva, T. F. Comportamento ingestivo e parâmetros fisiológicos de bovinos Sindi alimentados com teores crescentes de feno de erva-sal. Revista Brasileira de Ciências Agrárias, v.7, p.180-188, 2012. http://dx.doi.org/10.5039/agraria.v7i1a914

Perissinoto, M.; Moura, D. J. Determinação do conforto térmico de vacas leiteiras utilizando a mineração de dados. Revista Brasileira de Engenharia de Biossistemas, v.1, p.117-126, 2007.

Reece, W. O. Respiração nos mamíferos. Dukes: Fisiologia dos animais domésticos. 12. ed. Rio de Janeiro: Guanabara Koogan, 2006. p.103-134.

Robertshaw, D. Regulação da temperatura e o ambiente térmico. In: Reece, W. O. Dukes: Fisiologia dos animais domésticos. Rio de Janeiro: Guanabara Koogan, 2006. Cap. 55, 898p. 
Rodrigues, A. L.; Souza, B. B.; Pereira Filho, J. M. Influência do sombreamento e dos sistemas de resfriamento no conforto térmico de vacas leiteiras. Agropecuária Científica no Semiárido, v.6, p.14-22, 2010a.

Rodrigues, V. C.; Silva, I. J. O.; Vieira, F. C.; Nascimento, S. T. A correct enthalpy relationship as thermal comfort índex for livestock. International Journal of Biometeorology, v.55, p.455-459, $2010 \mathrm{~b}$. http://dx.doi.org/10.1007/s00484-010-0344-y

Sampaio, C. A. P.; Cardoso, C. O.; Souza, G. P. Temperaturas superficiais de telhas e sua relação com o ambiente térmico. Engenharia Agrícola, v.31, p.230-236, 2011.http://dx.doi.org/10.1590/S0100-69162011000200003

SAS - Statistical Analysis System. SAS companion for the microsoft windows environment. Version 8, Cary: SAS Institute, 2007. CD-Rom
Silva, J. J. N.; Montenegro, A. A. A.; Silva, E. F. F.; Fontes Júnior, R. V. P.; Silva, A. P. N. Variabilidade espacial de parâmetros de crescimento da mamoneira e de atributos físico-químicos em Neossolo. Revista Brasileira de Engenharia Agrícola e Ambiental, v.14, p.921-931, 2010. http://dx.doi.org/10.1590/ S1415-43662010000900003

Souza, B. B. da; Silva, I. J. O.; Mellace, E. M.; Santos, R. F. S.; Zotti, C. A.; Garcia, P. R. Avaliação do ambiente físico promovido pelo sombreamento sobre o processo termorregulatório em novilhas leiteiras. Agropecuária Científica no Semiárido, v.6, p.59-65, 2010.

Thom, E. C. The discomfort index. Weatherwise, v.12, p.57-59,1959. http://dx.doi.org/10.1080/00431672.1959.9926960 